\title{
FATORES QUE INFLUENCIAM O DESMAME NO RECÉM-NASCIDO PREMATURO
}

\author{
FACTORS WHICH INFLUENCE WEANING IN \\ PRETERM INFANT
}

\author{
Solange Maria de Saboia e Silva ${ }^{1}$ \\ Conceição Aparecida de Mattos Segre ${ }^{2}$
}

Silva, SMS e Segre CAM. Fatores que influenciam o desmame no recém-nascido prematuro. Rev. Bras. Cresc. e Desenv. Hum. 2010; (no prelo)

\section{Resumo:}

Objetivo: verificar os principais fatores que influenciam o desmame em prematuros nascidos em maternidade pública da cidade de São Paulo. Método: estudaram-se 89 prematuros de baixo peso acompanhados em ambulatório, no período de agosto de 2006 a maio de 2007. Esses prematuros foram divididos em dois grupos conforme a presença de desmame parcial (introdução de complemento lácteo por mamadeira) ou desmame total (interrupção do aleitamento materno). Foram estudadas variáveis maternas e das crianças, obtidas por meio de entrevista com a mãe e consulta a prontuários. Na análise estatística, utilizaram-se os testes $t$ (Student) e de correlação (Spearman). Adotou-se o nível de significância de 5\%. Resultados: a média de idade para o desmame parcial foi de 1,41 meses. Mães que realizaram ordenha durante a internação, ou que iniciaram ou retornaram ao trabalho, introduziram mamadeira mais tardiamente. Quanto menor o peso ao nascer e a idade gestacional, mais tardia a introdução da mamadeira. Quanto menor a idade de introdução da chupeta, mais precoce o desmame parcial. A média de idade para o desmame total foi de 2,93 meses. Mães que internaram na enfermaria canguru, ou que iniciaram ou voltaram ao trabalho, interromperam o aleitamento materno mais tardiamente. Considerações finais: os achados do presente trabalho apontam para a importância de fatores controláveis pela equipe de saúde na prevenção do desmame precoce em prematuros, como ordenha, metodologia canguru e orientação para o não uso de chupetas. Fatores não controláveis como peso ao nascer e trabalho materno, mostraram-se indicativos de maior permanência do aleitamento materno.

Palavras-chave: recém-nascido; prematuro; desmame.

1 Médica pediatra neonatologista do Hospital Municipal Maternidade-Escola de Vila Nova Cachoeirinha "Dr. Mário de Moraes Altenfelder Silva”, pós-graduada em Perinatologia pelo Instituto de Ensino e Pesquisa Albert Einstein. Mestre em Ciências da Saúde do Instituto de Assistência Médica ao Servidor Público Estadual (IAMSPE)

2 Livre-docente em Pediatria Neonatal pela Escola Paulista de Medicina, Universidade Federal de São Paulo (UNIFESP). Docente do programa de pós-graduação em Ciências da Saúde do Instituto de Assistência Médica ao Servidor Público Estadual (IAMSPE) Local do trabalho - Hospital Municipal Maternidade-Escola de Vila Nova Cachoeirinha "Dr. Mário de Moraes Altenfelder Silva”, São Paulo, Brasil

Correspondência para: Solange Maria de Saboia e Silva - Rua Dr. Diogo de Faria, 55 conj. 102 - Vila Clementino - São Paulo (SP) - Brasil - CEP 04037-000 - Telefone/fax: 5573-5813 - E-mail : solangesaboia@terra.com.br

Artigo baseado na dissertação de mestrado "Fatores que influenciam o desmame no recém-nascido/lactente prematuro", apresentada em dezembro de 2008 no Instituto de Assistência Médica ao Servidor Público Estadual (IAMSPE). 


\begin{abstract}
Objective: to verify the major factors influencing weaning in preterm infants born in a public maternity center in the city of Sao Paulo, Brazil. Method: a total of 89 low-birthweight preterm infants followed on an outpatient basis were studied from August 2006 to May 2007. These premature infants were divided into two groups according to the presence of partial weaning (introduction of supplementary bottle feeding) or full weaning (breastfeeding cessation). Maternal and infant variables obtained by means of interviews with the mothers and from the medical records were studied. The Student's $t$ test and Spearman rank correlation test were used for the statistical analysis. The significance level was set at 5\%. Results: mean age for partial weaning was 1.41 months. Mothers who pumped during hospitalization or who started working or returned to work started bottle feeding later. The lower the birth weight and the gestational age, the later bottle feeding was started. The lower the age at pacifier introduction, the earlier the partial weaning. Mean age for full weaning was 2.93 months. Mothers hospitalized in the kangaroo mother care unit, or those who started working or returned to work, stopped breastfeeding later. Final Considerations: the findings of the present study point to the importance of factors that can be controlled by the health care team - such as breast milk pumping, the kangaroo mother care method, and advice not to use pacifiers - in the prevention of early weaning in preterm infants. Uncontrollable factors such as birth weight and maternal work were predictive of longer breastfeeding.
\end{abstract}

Key words: infant; premature; weaning.

\section{INTRODUÇÃO}

Desde os primórdios da Neonatologia, o recém-nascido $(\mathrm{RN})$ prematuro constitui um desafio e os avanços nessa área são frutos da necessidade de diminuir a morbidade e a mortalidade desses RN e a evolução tecnológica permitiu-lhes maior sobrevida ${ }^{1}$. Pesquisas sobre o desenvolvimento neuro-comportamental dessas crianças mostraram a necessidade de cuidados integrados e recuperação do aleitamento materno $(\mathrm{AM})^{2}$ por meio do resgate da presença materna nas unidades neonatais ${ }^{3,4}$.

Ao conceito da década de 80 de que o AM seria benéfico devido a fatores psicológicos, somou-se o conhecimento científico dos anos 90 sobre sua ação protetora, levando à diminuição da incidência de enterocolite necrosante, infecções hospitalares e do tempo de internação ${ }^{5,6}$. Esses benefícios continuam após a alta, com diminuição da morbidade no primeiro ano de vida e das taxas de desnutrição e mortalidade infantil. ${ }^{5}$ Pesquisas também mostram evidências de ação benéfica no desenvolvimento neurológico e aspectos cognitivos a médio e longo prazo ${ }^{7}$.

Apesar desses fatos, a incidência de AM é menor nesses RN. ${ }^{7}$ Estudo revelou que das 87 mães de prematuros que planejavam realizar o AM, apenas $34 \%$ continuaram a lactação até 40 semanas de idade corrigida e apenas 14\% amamentavam o lactente ao seio pelo menos uma vez ao dia aos quatro meses de idade corrigida $^{8}$. O Brasil, no entanto, apresenta estatísticas melhores. Pesquisa em hospital com metodologia canguru mostrou freqüência de 94,6\% para o AM e $84,4 \%$ para aleitamento materno exclusivo (AME) ${ }^{2}$ no momento da alta hospitalar 7 . Outra, realizada com 72 mães de prematuros de muito baixo peso, mostrou au- 
mento nas taxas de AME (19,5\%) e AM $(80,5 \%)$ no grupo que recebeu orientação adicional $^{9}$.

Estudos sobre o desmame em RN a termo após a alta hospitalar mostraram que os principais fatores relacionados foram impressão materna de pouco leite, leite fraco, problemas com a pega, uso de chupetas, idade e escolaridade materna. No entanto, poucos estudos focalizaram os fatores de desmame em prematuros $^{10-13}$.

Apesar dos avanços das últimas décadas, ainda convivemos com dúvidas sobre o desmame nos prematuros, justificando-se a ampliação de pesquisas nessa área ${ }^{14}$. Assim, o objetivo é verificar as principais variáveis maternas e do RN/lactente prematuro que influenciam o desmame durante a internação ou após a alta hospitalar e identificar fatores passíveis de intervenção para sua prevenção.

\section{MÉTODO}

Estudo descritivo observacional série de casos em hospital maternidade de nível terciário da rede pública municipal. A instituição em questão fica localizada na periferia da cidade de São Paulo.

Participaram da pesquisa mães e RN/ lactentes prematuros, atendidos no ambulatório no período de agosto de 2006 a maio de 2007. Esses prematuros estiveram internados na Unidade Neonatal de Alto Risco, exceto um caso que permaneceu no Alojamento Conjunto. Todos os RN/lactentes incluídos no estudo foram examinados pelo pesquisador (SS) e tinham idade gestacional menor que 37 semanas, peso ao nascer menor que $2500 \mathrm{~g}$ e apresentaram desmame parcial ou total antes do sexto mês de vida. As mães incluídas concordaram com os termos da pesquisa e assinaram o termo de consentimento livre e esclarecido. Foram excluídos os RN/lactentes portadores de malformações e/ou suspeitos de síndrome ge- nética, neuropatas, mães portadoras do vírus da imunodeficiência humana, doença materna que impedisse o AM, óbito materno, adoção, segundo gemelar e aqueles com dados insuficientes.

De agosto de 2006 a maio de 2007 foram matriculados 1231 prematuros no ambulatório de acompanhamento, sendo 867 atendidos pelo pesquisador (SS). Devido à freqüência de desmame precoce em prematu$\operatorname{ros}^{8,9}$, estimou-se, em função do número de variáveis envolvidas e da freqüência de desmame parcial e total, que a amostra deveria ser composta por 60 crianças, com número mínimo de 30 crianças. No período estudado, apenas 32 crianças apresentaram desmame total. As mães e seus prematuros foram selecionados aleatoriamente segundo a ordem de atendimento ambulatorial, respeitando-se os critérios de inclusão e exclusão. A perda amostral foi de 778 crianças que não se adequavam a esses critérios. A amostra final foi composta por 89 prematuros divididos em dois grupos. O grupo 1 contou com 81 crianças e o grupo 2 com 32, sendo 24 provenientes do grupo 1. A amostra do grupo 2 foi formada após a do grupo 1 , devido à necessidade de se completar o número mínimo exigido para o estudo. Oito crianças do grupo 2, portanto, não foram incluídas no grupo 1, e apresentaram-se à consulta já em desmame total. Variáveis maternas e das crianças foram obtidas dos prontuários e da entrevista materna. RN/ lactentes que receberam leite materno e complemento lácteo com mamadeira até o sexto mês, foram considerados em desmame parcial (grupo 1) e aqueles que interromperam o AM e passaram a receber somente mamadeira (grupo 2), foram considerados em desmame total.

Foram estudadas variáveis maternas demográficas, socioeconômicas, relacionadas aos antecedentes obstétricos, à assistência pósnatal imediata, às intercorrências no período gestacional e puerperal e a aspectos 
psicossociais. Dentre as variáveis socioeconômicas, analisaram-se renda familiar, escolaridade materna e paterna, tabagismo, trabalho materno e apoio. Foi considerado apoio quando houve pessoa que incentivasse o $\mathrm{AM}$ e/ou ajudasse nos afazeres domésticos. Dentre os aspectos psicossociais, analisaram-se impressão materna de pouco leite, leite fraco e se o RN/lactente mamava bem. Considerou-se que mamava bem, quando a mãe referia boa pega e sucção adequada ${ }^{10,12,15-16}$.

Dentre as variáveis relacionadas à assistência pós-natal imediata, investigaram-se a ocorrência de mastite, a prática de ordenha durante o período de internação do RN, internação na enfermaria canguru e informações sobre AM fornecidas pela equipe. ${ }^{10,12,15-16}$

Com relação ao RN/lactente, foram analisadas variáveis demográficas, relacionadas ao nascimento, ao período de internação e período pós-alta. Além da morbidade foram analisados dias de utilização de sonda oro/nasogástrica, intubação orotraqueal e dias de internação. Nesse período, avaliou-se a utilização de mamadeira e essa variável só foi analisada em relação ao desmame total. No período pós-alta foi estudada idade de introdução da chupeta, número de consultas ambulatoriais, ocorrência de internações, idade de introdução da mamadeira (desmame parcial) e idade da interrupção do AM (desmame total). Foram considerados distúrbios metabólicos, os do cálcio e magnésio. Hidroeletrolíticos, os do sódio, potássio e do equilíbrio ácido-base. As variáveis analisadas foram selecionadas baseadas nos critérios de freqüência e classificação usualmente descritos na literatura ${ }^{10,12,15-16}$. Os dados coletados foram anotados em planilhas Excel.

Os testes estatísticos utilizados para a análise das variáveis foram o teste $t$ (Student) e o de correlação de Spearman $\left(\mathrm{r}_{\mathrm{s}}\right)$. Adotou-se o nível de significância de $5 \%$.

O presente estudo foi aprovado pelo Comitê de Ética em Pesquisa da instituição onde o trabalho foi realizado (parecer 02/2006).

\section{RESULTADOS}

Quanto ao desmame parcial, a média de idade materna encontrada foi 26,58 anos (DP $\pm 7,11$ ) e a renda familiar média de 778,88 reais (DP $\pm 520,01)$. Para a escolaridade materna e paterna, obtiveram-se médias de 8,01 (DP \pm $2,81)$ e 7,52 ( $\mathrm{DP} \pm 3,35)$ anos respectivamente (tabela 1). Em relação aos RN/lactentes, a

Tabela 1: Características maternas, paternas e dos recém-nascidos/lactentes dos grupos 1 e 2

\begin{tabular}{|c|c|c|c|c|}
\hline & $\mathrm{N}$ & Média & $\begin{array}{l}\text { Desvio } \\
\text { Padrão }\end{array}$ & Mediana \\
\hline \multicolumn{5}{|l|}{ Grupo 1} \\
\hline \multicolumn{5}{|l|}{ Desmame } \\
\hline parcial (meses) & 81 & 1,41 & 1,09 & .. \\
\hline Idade materna & 81 & 26,58 & 7,11 & .. \\
\hline $\begin{array}{l}\text { Renda familiar } \\
\text { Escolaridade }\end{array}$ & 76 & 778,88 & 520,01 & . \\
\hline materna & 81 & 8,01 & 2,81 & .. \\
\hline Escolaridade & & & & \\
\hline $\begin{array}{c}\text { paterna } \\
\text { Peso }\end{array}$ & 79 & 7,52 & 3,35 & .. \\
\hline ao nascer (g) & 81 & $1.590,74$ & 432,07 &. \\
\hline Idade gestacional & 81 & .. & .. & 33,0 \\
\hline \multicolumn{5}{|l|}{ Grupo 2} \\
\hline \multicolumn{5}{|l|}{ Desmame } \\
\hline total (meses) & 32 & 2,93 & 1,54 & .. \\
\hline Idade materna & 32 & 24,59 & 6,21 & .. \\
\hline $\begin{array}{l}\text { Renda familiar } \\
\text { Escolaridade }\end{array}$ & 28 & 921,43 & 705,82 & .. \\
\hline $\begin{array}{c}\text { materna } \\
\text { Escolaridade }\end{array}$ & 32 & 8,81 & 2,56 & .. \\
\hline paterna & 31 & 7,45 & 2,78 & .. \\
\hline $\begin{array}{c}\text { Peso ao } \\
\text { nascer (gramas) }\end{array}$ & 32 & $1.542,97$ & 429,34 & .. \\
\hline $\begin{array}{l}\text { Idade gestacional } \\
\text { (semanas) }\end{array}$ & 32 & .. & .. & 33,5 \\
\hline
\end{tabular}

amostra foi constituída por $43 \mathrm{RN} /$ lactentes do sexo feminino e 38 do sexo masculino, correspondendo a $53,1 \%$ e $46,9 \%$ respectivamente. O peso ao nascer variou entre $750 \mathrm{~g}$ a 2.490g, com média de 1.590,74g ( $\mathrm{DP} \pm 432,07)$. A idade gestacional variou entre 26 e 36,8 semanas com mediana de 33,0 semanas (tabela 1). O Apgar de primeiro minuto esteve entre um e nove com mediana de 8,0 e o de quinto minuto entre seis e dez com mediana de 9,0. 
O teste de correlação entre as variáveis maternas quantitativas e o desmame parcial, não mostrou resultados estatisticamente significativos. Em relação às variáveis maternas qualitativas, obtiveram-se resultados significativos para ordenha $(t=2,843, \mathrm{p}=0,007)$, impressão de pouco leite $(t=2,405, \mathrm{p}=0,022) \mathrm{e}$ trabalho materno $(t=4,110, \mathrm{p}=0,001)$. Mães que realizaram ordenha e que iniciaram ou voltaram ao trabalho, introduziram mamadeira mais tardiamente. Mães que tiveram impressão de pouco leite, introduziram mamadeira mais precocemente (tabela 2).
As variáveis quantitativas relacionadas ao RN/lactente, mostraram correlação negativa entre o peso ao nascer e o desmame parcial $\left(r_{s}=-0,524, p<0,001\right)$. Quanto menor o peso, mais tardia a introdução da mamadeira. O mesmo tipo de correlação foi encontrado para a idade gestacional $\left(\mathrm{r}_{\mathrm{s}}=-0,454, \mathrm{p}<0,001\right)$. Quanto menor a idade gestacional, mais tardia a introdução da mamadeira. Houve correlação positiva entre o desmame parcial e os dias de intubação orotraqueal $\left(r_{S}=0,421\right.$, $\mathrm{p}<0,001)$, dias de utilização da sonda orogástrica $\left(r_{\mathrm{s}}=0,430, \mathrm{p}<0,001\right)$ e dias de

Tabela 2: Médias da idade do desmame parcial, teste $t$ e nível de significância da comparação entre os dois grupos (Sim e Não) das variáveis qualitativas maternas e dos recém-nascidos/ lactentes do grupo 1

\begin{tabular}{|c|c|c|c|c|c|c|}
\hline \multirow{3}{*}{ Variáveis } & \multicolumn{5}{|c|}{ Desmame Parcial (meses) } & \multirow{3}{*}{$\mathrm{p}$} \\
\hline & & Não & & Sim & $t$ & \\
\hline & média & desvio padrão & média & desvio padrão & $l$ & \\
\hline \multicolumn{7}{|l|}{ Maternas } \\
\hline Ordenha & 0,92 & 0,45 & 1,46 & 1,11 & 2,843 & $0,007(*)$ \\
\hline Pouco Leite & 1,83 & 1,33 & 1,14 & 0,80 & 2,405 & $0,022(*)$ \\
\hline Trabalho materno & 1,08 & 0,62 & 2,75 & 1,48 & 4,110 & $0,001(*)$ \\
\hline \multicolumn{7}{|l|}{ Recém-nascidos/lactentes } \\
\hline Apnéia & 1,26 & 1,09 & 2,11 & 0,82 & 2,739 & $0,008(*)$ \\
\hline Hemorragia intracraniana & 1,32 & 1,06 & 2,39 & 1,03 & 2,554 & $0,013(*)$ \\
\hline Broncodisplasia pulmonar & 1,30 & 1,08 & 1,98 & 1,00 & 1,998 & 0,049 \\
\hline
\end{tabular}

(*) p significativo, $\mathrm{p}<5$

internação $\left(\mathrm{r}_{\mathrm{S}}=0,528, \mathrm{p}<0,001\right)$. Quanto maior o valor dessas variáveis, mais tardia a introdução da mamadeira. Encontrou-se correlação positiva entre o desmame parcial, a idade de introdução da chupeta $\left(\mathrm{r}_{\mathrm{s}}=0,653, \mathrm{p}<\right.$ $0,001)$ e o número de consultas ambulatoriais $\left(\mathrm{r}_{\mathrm{s}}=0,397, \mathrm{p}<0,001\right)$. Quanto mais tardia a introdução da chupeta e quanto maior o número de consultas ambulatoriais, mais tardia a introdução da mamadeira. A correlação entre o peso ao nascer e a idade do desmame parcial, pode ser observada na figura 1 , que mostra o gráfico correspondente com a respectiva reta de regressão.Os resultados descritos, podem ser verificados na tabela 3.

Quanto às variáveis qualitativas relacionadas ao RN/lactente, apnéia $(t=2,739, \mathrm{p}=$
Figura 1: Scatterplot do desmame parcial e peso ao nascer, com a respectiva reta de regressão $(\mathrm{Y}=2,609-0,001 \mathrm{x}$ peso nascer; $\mathrm{p}=0,007 ; \mathrm{r}^{2}=0,09$ )

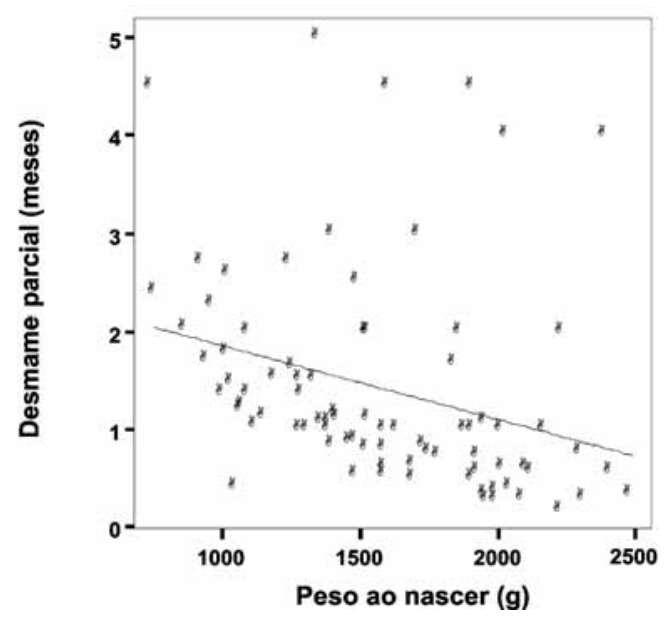


Tabela 3: Coeficientes de correlação entre os desmames e as variáveis quantitativas dos recém-nascidos/lactentes dos grupos 1 e 2

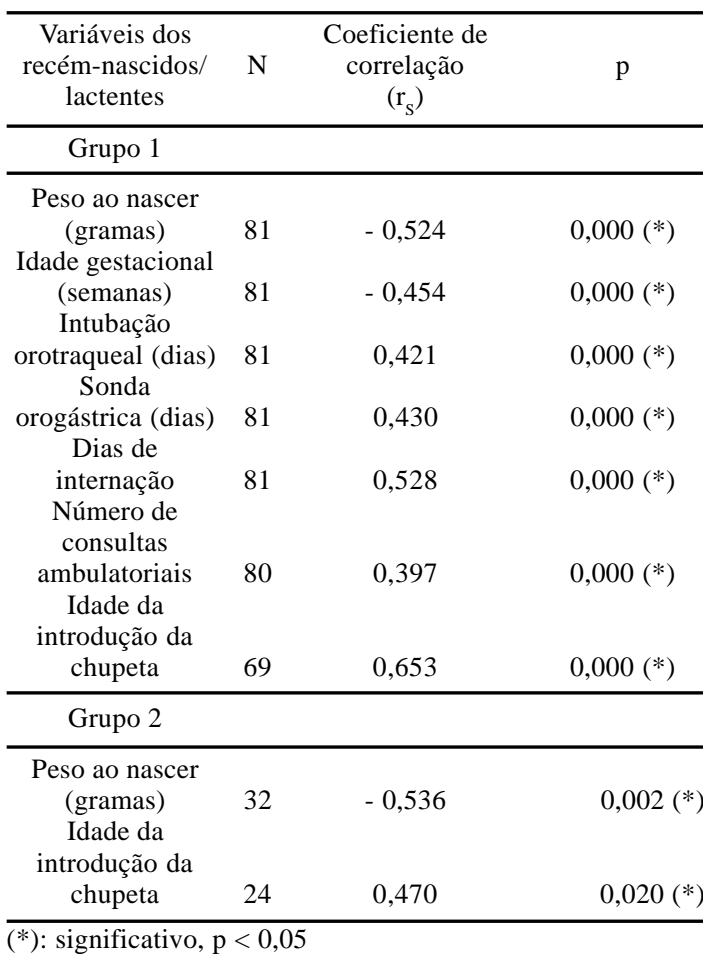

$0,008)$, hemorragia intracraniana $(t=2,554$, $\mathrm{p}=0,013)$ e broncodisplasia pulmonar $(t=$ $1,998, p=0,049$ ), mostraram diferenças significativas. O desmame parcial foi mais tardio naqueles que apresentaram as características citadas. (tabela 2)
Quanto ao desmame total, a média da idade materna foi de 24,59 anos (DP $\pm 6,21$ ) e a renda familiar média de 921,43 reais (DP $\pm 705,82$ ). Para a escolaridade materna e paterna, obteve-se 8,81 (DP $\pm 2,56$ ) e 7,45 (DP $\pm 2,78$ ) anos respectivamente (tabela 1 ). Quinze RN/lactentes eram do sexo feminino e 17 do sexo masculino, correspondendo a $46,9 \%$ e $53,1 \%$ respectivamente. O peso ao nascer variou de $750 \mathrm{~g}$ a $2.245 \mathrm{~g}$, com média de $1.542,97 \mathrm{~g}(\mathrm{DP} \pm 429,34)$. A idade gestacional variou entre 26,0 e 36,50 semanas com mediana de 33,5 semanas (tabela 1). O Apgar de primeiro minuto variou entre um e nove, com mediana de 7,0. Para o Apgar de quinto minuto encontraram-se valores entre seis e dez, com mediana de 9,0.

As variáveis maternas que apresentaram resultados significativos foram internação na enfermaria canguru $(t=2,211, p=0,035)$, trabalho materno $(\mathrm{t}=3,008, \mathrm{p}=0,005)$ e apoio $(t=2,509, p=0,018)$. Mães que foram internadas nessa enfermaria e aquelas que iniciaram ou retornaram ao trabalho, realizaram o desmame total mais tardiamente. As que relataram ter recebido apoio, realizaram o desmame total mais precocemente. (tabela 4)

Das variáveis relacionadas ao RN/ lactente, encontraram-se resultados significa-

Tabela 4: Médias da idade do desmame total, teste $t$ e nível de significância da comparação entre os dois grupos (Sim e Não) das variáveis qualitativas maternas e dos recém-nascidos/lactentes do grupo 2

\begin{tabular}{ccccccc}
\hline \multicolumn{7}{c}{ Desmame Total (meses) } \\
\hline $\begin{array}{c}\text { Variáveis } \\
\text { Maternas }\end{array}$ & média & $\begin{array}{c}\text { Não } \\
\text { desvio padrão }\end{array}$ & média & $\begin{array}{c}\text { Sim } \\
\text { desvio padrão }\end{array}$ & $t$ & $\mathrm{p}$ \\
\hline $\begin{array}{c}\text { Intermação } \\
\text { na enfermaria } \\
\text { canguru }\end{array}$ & 2,69 & 1,48 & 4,25 & 1,25 & 2,211 & $0,035\left(^{*}\right)$ \\
\hline Trabalho matermo & 2,58 & 1,38 & 4,35 & 1,31 & 3,008 & $0,005\left(^{*}\right)$ \\
\hline Apoio & 3,52 & 1,46 & 2,21 & 1,41 & 2,509 & $0,018\left(^{*}\right)$ \\
\hline
\end{tabular}

$(*)$ p significativo, $\mathrm{p}>5$ 
tivos para peso ao nascer $\left(\mathrm{r}_{\mathrm{s}}=-0,536, \mathrm{p}=\right.$ $0,002)$ e utilização de chupeta $\left(r_{s}=0,470, p=\right.$ $0,020)$. Quanto menor o peso e quanto maior a idade de introdução da chupeta, mais tardio o desmame total (tabela 3). A correlação entre o peso ao nascer e desmame total, pode ser observada na figura 2. As demais variáveis não apresentaram resultados significativos.

Figura 2: Scatterplot do desmame total e peso ao nascer, com a respectiva reta de regressão $(\mathrm{Y}=5,95-0,002 \mathrm{x}$ peso nascer; $\mathrm{p}=0,001$; $\left.\mathrm{r}^{2}=0,54\right)$.

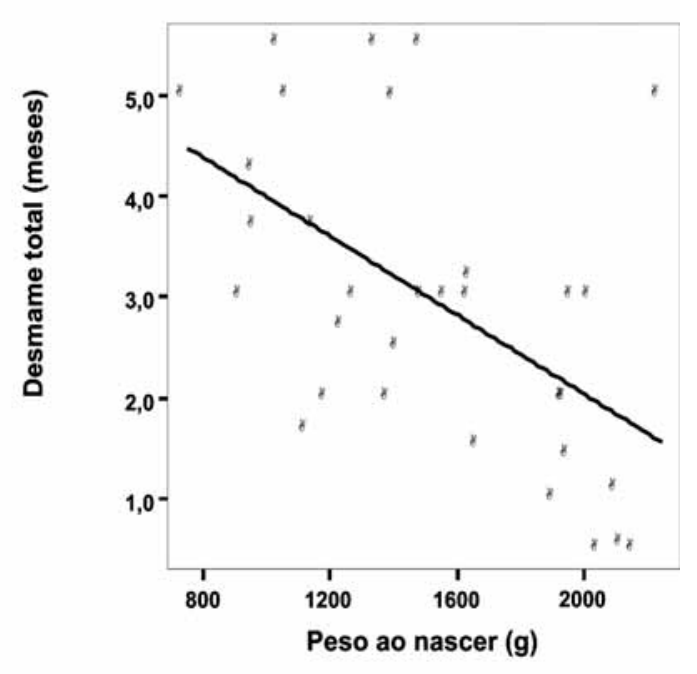

\section{DISCUSSÃO}

\section{Desmame parcial (grupo 1)}

No presente estudo, as variáveis quantitativas maternas estudadas não mostraram diferenças significativas, de acordo com o que se encontra na literatura ${ }^{11,17}$.

Dentre as variáveis qualitativas maternas, no presente estudo, a prática da ordenha prorrogou a introdução da mamadeira, provavelmente relacionada ao maior volume de leite produzido. A retirada de leite por meio da ordenha permite a manutenção da lactância mesmo em mães cujos prematuros não conseguiram amamentar nas primeiras duas semanas após o nascimento e influen- cia o volume produzido nas semanas que se seguem ao parto ${ }^{18-19}$.

A impressão materna de pouco leite é registrada na literatura, como uma das principais causas para a introdução da mamadeira ou para a interrupção do AM em RN termo ou prematuro. Na maioria das vezes foi atribuída a impressões subjetivas, à falta de informação materna ou interpretada como um reflexo de conceitos higienistas sobre o $\mathrm{AM}^{20-21}$. Por outro lado, as pesquisas demonstraram que mães de prematuros têm risco 2,81 vezes maior para produção inadequada de leite e que a percepção materna de pouco leite estava associada à menor produção ${ }^{18-19}$. O presente estudo também demonstrou que a impressão materna de pouco leite levou ao desmame parcial mais precoce, e não se pôde excluir também que fosse devido à diminuição efetiva do volume de leite produzido ${ }^{15,18-19}$.

Quanto ao trabalho materno, a maioria das pesquisas mostra maior risco para a interrupção do $\mathrm{AME}^{22-24}$. Os resultados do presente estudo demonstraram que mães trabalhadoras amamentaram por mais tempo e introduziram mamadeira mais tardiamente. Vale ressaltar que não foi coletada a informação sobre a época do início, tipo de trabalho exercido e horas diárias trabalhadas. Mesmo em trabalhadoras formais, horários flexíveis proporcionaram um maior período de $\mathrm{AM}^{24}$. Outro fator é que a maioria dos estudos sobre trabalho materno foi realizada incluindo crianças em AME e a presente pesquisa excluiu-as. No entanto, os dados obtidos sugerem que mães trabalhadoras se esforçaram para prolongar o AM talvez por preocupação com a saúde dos seus filhos prematuros ${ }^{25}$.

Das características relacionadas ao RN/ lactente, o peso de nascimento e a idade gestacional têm sido abordados por pesquisadores, não sendo encontrados resultados significativos ${ }^{8,17}$. No presente estudo, houve correlação negativa entre o peso e idade gestacional e o desmame parcial. Assim, quanto 
menor o peso ao nascer e a idade gestacional, mais tardia a introdução da mamadeira. Esses resultados podem ter sido influenciados pelo início mais tardio da alimentação oral devido à morbidade relacionada ou à preocupação com sua saúde e ganho de peso, fator assinalado por Javorsky ${ }^{25}$.

O tempo de intubação e de utilização de sonda orogástrica não têm sido analisados isoladamente, estando relacionados de forma indireta à morbidade neonatal ${ }^{8,16}$. No presente estudo, esses fatores foram pesquisados isoladamente e encontrou-se que quanto maior o tempo de intubação e de sonda orogástrica, mais tardiamente foi introduzida a mamadeira. Esses resultados provavelmente foram também influenciados pelo peso ao nascimento e idade gestacional, que quanto menores, mais tardio o desmame parcial, corroborando os dados obtidos para essas variáveis no presente estudo.

O número de dias de internação tem sido considerado barreira à implementação do AM, mas pode-se inferir que essa circunstância irá modificar as práticas de alimentação dessas crianças e influenciar no desmame, seja ele parcial ou total ${ }^{8,15}$. No presente estudo, encontrou-se resultado discordante da literatura quanto à permanência no hospital. Quanto maior o período de permanência, mais tardia a introdução da mamadeira. Uma das hipóteses para os resultados encontrados pode estar relacionada à aplicação da metodologia canguru, propiciando a participação da mãe e da família na recuperação de seu prematuro ${ }^{4}$. Essa hipótese é corroborada pela literatura que encontrou freqüências maiores de AME em lactentes prematuros que haviam participado da metodologia $^{22}$. Além disso, nos prematuros que fizeram parte do presente trabalho e que ficaram por mais tempo internados, o acompanhamento fonoaudiológico foi realizado em todas as mamadas, resultando em ação individualizada e constituindo momento de esclarecimento e suporte a essas mães ${ }^{4,7,26}$. O dado encontrado de- monstrou a importância de uma política institucional adequada e da ação de equipe multidisciplinar no difícil período de transição da alimentação enteral ao seio materno ${ }^{4,7}$.

Os efeitos da utilização de chupetas têm sido investigados por diversos autores e a maioria dos estudos aponta a chupeta como fator envolvido no processo de desmame parcial ou total $^{13,17,23,27}$. No presente estudo, essa variável foi analisada e demonstrou-se que quanto mais tardia a introdução da chupeta, mais tardia a introdução da mamadeira. Por constituir hábito cultural arraigado nos diversos grupos estudados, faz-se necessária orientação adequada às mães e familiares quanto à sua utilização e os efeitos negativos sobre o $\mathrm{AM}^{27}$.

A importância do acompanhamento ambulatorial no período pós-alta tem sido demonstrada por algumas pesquisas que analisaram as freqüências de AM e a incidência do desmame parcial quando esse acompanhamento foi realizado. ${ }^{9,22}$ Os dados do presente estudo estão de acordo com os da literatura e mostram a importância da equipe de saúde e da continuidade no suporte a essas mães após a alta hospitalar ${ }^{9,22}$. A influência da morbidade neonatal sobre o desmame, tem sido abordada em relação ao desmame total e não tem apresentado resultados significativos ${ }^{8,16}$. No presente estudo, a morbidade foi analisada em relação ao desmame parcial e encontraram-se resultados significativos para broncodisplasia, apnéia e hemorragia intracraniana. Assim, nos RN/lactentes que apresentaram esses quadros, a introdução da mamadeira foi mais tardia. Esses dados podem ter sido influenciados pelo estabelecimento mais tardio da alimentação enteral, mas provavelmente estiveram relacionados aos efeitos do peso e idade gestacional que quanto menores adiaram a introdução da mamadeira.

Algumas pesquisas evidenciaram aumento na freqüência de aleitamento para aquelas mães que receberam algum tipo de apoio, seja durante o pré-natal, internação ou período 
pós-alta ${ }^{4,5}$. No presente estudo, o apoio foi investigado em relação ao período pós-alta e mães que receberam "apoio", conceituado de acordo com a interpretação materna, realizaram o desmame mais cedo. Isso leva à reflexão de que essas mães poderiam estar em ambiente social e familiar inadequado para a contenção e ajuda às suas dificuldades ${ }^{20}$.

\section{Desmame Total - Grupo 2}

A literatura tem assinalado os benefícios da metodologia canguru em relação à duração do $\mathrm{AM}^{4}$. No presente trabalho, mães que ficaram internadas junto aos seus prematuros, participando da metodologia canguru, interromperam o AM mais tardiamente. Esses resultados estão de acordo com os da literatura e demonstram a importância do método para esse grupo de crianças ${ }^{4,22}$.

Quanto ao trabalho materno, da mesma forma como foi visto para o desmame parcial, em desacordo com a literatura, no presente estudo, mães trabalhadoras interromperam o AM mais tardiamente do que as não trabalhadoras. Apesar de não ter sido investigado o tipo de trabalho exercido, o resultado chama a atenção e como descrito, pode significar um esforço maior dessas mães relacionado à preocupação com a saúde de seus prematuros ${ }^{25}$.

Em relação ao peso de nascimento, os dados da literatura demonstram que o peso ao nascer não aumenta o risco para a interrupção do AM além do esperado para a prematurida$\mathrm{de}^{8,16}$. No presente estudo, o peso ao nascer mostrou correlação negativa com o desmame total. Quanto menor o peso ao nascer mais tardio o desmame. Essa característica sugere que mães de prematuros menores se esforçam para superar dificuldades e prolongar o AM por mais tempo ${ }^{25}$.

As pesquisas realizadas fornecem fortes evidências sobre a influência da chupeta nos processos de desmame parcial e total ${ }^{17,23,27}$. O presente estudo analisou a época da introdução da chupeta após a alta hospitalar e ve- rificou que quanto mais tardia a introdução da chupeta, mais tardia a interrupção do AM, da mesma forma com o ocorrido em relação ao desmame parcial. Outrossim, a necessidade de utilização da chupeta para estimulação da sucção não nutritiva nessas crianças ${ }^{28}$, ou como fator protetor para a síndrome da morte súbita do lactente que tem sido alvo de preocupação por parte da comunidade científica ${ }^{29}$, a equipe de saúde deve levar em consideração o risco de desmame que implica na utilização da chupeta.

Os resultados do presente trabalho apontam para a tendência dessas mães a manterem o AM por mais tempo devido à preocupação com a saúde e recuperação de seus filhos ${ }^{25}$. O conhecimento acerca dessa preocupação materna com prematuros de maior risco, pode ser de fundamental importância para a equipe de saúde quanto ao incentivo e apoio ao AM nessas crianças.

Desta maneira, a prática da ordenha materna prorrogou o tempo para a introdução de mamadeira; o trabalho materno influenciou a época do desmame total e parcial. Mães trabalhadoras introduziram mamadeira mais tardiamente e amamentaram seus prematuros por mais tempo; a impressão materna de pouco leite, contribuiu para a introdução mais precoce da mamadeira; aquelas que receberam apoio de familiares ou amigos, efetuaram o desmame total mais precocemente; quanto à permanência na enfermaria canguru, o desmame total foi mais tardio nas mães que participaram da metodologia; o menor peso ao nascimento esteve relacionado ao desmame parcial e total, ambos ocorrendo de forma mais tardia em RN/ lactentes que nasceram com pesos menores; 0 uso da chupeta esteve relacionado ao desmame parcial e total e os resultados fornecem evidências sobre a influência desse hábito no desmame dessas crianças.

Assim, ficou evidenciado, pelo presente trabalho, que há fatores controláveis pela equipe de saúde que podem influenciar para 
que seja evitado o desmame precoce em prematuros (prática da ordenha materna, participação na metodologia canguru e a orientação para o não uso de chupetas) bem como fatores não controláveis pela equipe de saúde (peso ao nascer e trabalho materno), contudo, seu conhecimento é importante para propiciar da-

\section{REFERENCIAS}

1. Raju TNK. From infant hatcheries to intensive care: some highlights of the century of neonatal medicine. In: Fanaroff AA, Martin RJ. Neonatal-perinatal medicine: diseases of the fetus and infants. 7th ed. St. Louis: Mosby; 2002. p.2-16.

2. World Health Organization. Division of child health and development. Indicators for assessing breastfeeding practices. Geneva: OMS;1991. 16p.

3. Als H. Neurobehavioral development of the preterm infant. In: Fanaroff AA, Martin RJ. Neonatal-perinatal medicine: diseases of the fetus and infants. 7th ed. St. Louis: Mosby; 2002. p.947-71.

4. Brasil. Ministério da Saúde. Secretaria de Políticas de Saúde. Área técnica da saúde da criança. Atenção humanizada ao recémnascido de baixo peso-Método Canguru. Brasília: Ministério da Saúde; 2001.

5. Gouvêa L. The role of maternal milk in the prevention of diseases. einstein. 2005;3(2):119-22.

6. Segre CAM. Perinatologia fundamentos e prática. São Paulo: Savier; 2002. Capítulo 3 , Prioridades Obstétricas. Enterocolite necrosante.p.207-13.

7. do Nascimento MB, Issler $\mathrm{H}$. [Breastfeeding in premature infants: inhospital clinical management] J Pediatr (Rio J). 2004;80(5 Suppl):S163-72. Portuguese.

8. Furman L, Minich N, Hack M. Correlates of lactation in mothers of very low birth dos à equipe no sentido de esclarecer e incentivar as mães na manutenção do AM.

Um aspecto limitante do presente estudo refere-se aos dados sobre a época dos desmames, que podem ter sofrido influência da memória materna já que não foi utilizado o padrão de alimentação nas últimas 24 horas.

weight infants. Pediatrics. 2002; 109(4): e 57.

9. Santoro Junior W, Martinez FE. Effect of intervention on the rates of breastfeeding of very low birth weight newborns. J Pediatr (Rio J). 2007;83(6):541-46.

10. Caldeira AP, Goulart EM. [Breastfeeding in Montes Claros, Minas Gerais: a representative sample study] J Pediatr (Rio J). 2000;76(1):65-72. Portuguese.

11. Soares ME, Giugliani ER, Braun ML, Salgado AC, de Oliveira AP, de Aguiar PR. [Pacifier use and its relationship with early weaning in infants born at a Child-Friendly Hospital] J Pediatr (Rio J). 2003;79(4):309-16. Portuguese.

12. Callen J, Pinelli J. A review of the literature examining the benefits and challenges, incidence and duration, and barriers to breastfeeding in preterm infants. Adv Neonatal Care. 2005;5(2):72-88.

13. França MC, Giugliani ER, Oliveira LD, Weigert EM, Santo LC, Köhler CV, et al. Bottle feeding during the first month of life: determinants and effect on breastfeeding technique. Rev Saude Publica [Internet]. 2008 Aug [cited 2008 Sep 25;42(4):607-14. Available from: http://www.scielosp.org/pdf/rsp/ 2008nahead/6206.pdf

14. Hill PD, Ledbetter RJ, Kavanaugh KL. Breastfeeding patterns of low-birth-weight infants after hospital discharge. J Obstet Gynecol Neonatal Nurs. 1997;26(2):189-97.

15. Hill PD, Aldag JC, Zinaman M, Chatterton RT. Predictors of preterm infant feeding 
methods and perceived insufficient milk supply at week 12 postpartum. J Hum Lact. 2007;23(1):32-8.

16. Flacking R, Wallin L, Ewald U. Perinatal and socioeconomic determinants of breastfeeding duration in very preterm infants. Acta Paediatr. 2007;96(8):112630.

17. Benevenuto de Oliveira MM, Thomson Z, Vannuchi MT, Matsuo T. Feeding patterns of Brazilian preterm infants during the first 6 months of life, Londrina, Parana, Brazil. J Hum Lact. 2007;23(3):269-74.

18. Hill PD, Aldag JC. Milk volume on day 4 and income predictive of lactation adequacy at 6 weeks of mothers of nonnursing preterm infants. J Perinat Neonatal Nurs. 2005;19(3):273-82.

19. Hill PD, Aldag JC, Chatterton RT, Zinaman M. Comparison of milk output between mothers of preterm and term infants: the first 6 weeks after birth. J Hum Lact. 2005 Feb;21(1):22-30.

20. Almeida JAG, Novak FR. Breastfeeding: a nature-culture hybrid. J Pediatr (Rio J). 2004;80(5 Supl):S119-S125. Portuguese.

21. Giugliani ER. [Common problems during lactation and their management] J Pediatr (Rio J). 2004;80(5 Suppl):S147-54.

22. Alves AML, Silva EHAA, Oliveira AC. Desmame precoce em prematuros participantes do Método Mãe Canguru. Rev Soc Bras Fonoaudiol. 2007;12(1):23-2.

23. Vannuchi MTO,Thomson Z, Escuder MML, Tacla MTGM, Vezozzo KMK, Castro LMCP, et al. Perfil do aleitamento materno em menores de um ano no
Município de Londrina, Paraná. Rev Bras Saúde Matern Infant. 2005;5(2):155-62.

24. Rea MF, Venâncio SI, Batista LE, dos Santos RG, Greiner T. [Possibilities and limitations of breast-feeding among formally employed women] Rev Saude Publica. 1997;31(2):149-56. Portuguese.

25. Javorski M, Caetano LC, Vasconcelos MGL, Leite AM, Scochi CGS. As representações sociais do aleitamento materno para mães de prematuros em unidade de cuidado canguru. Rev Latinoam Enferm. 2004;12(6):890-8.

26. Sanches MT. [Clinical management of oral disorders in breastfeeding] J Pediatr (Rio J). 2004;80(5 Suppl):S155-62.

27. Victora CG, Behague DP, Barros FC, Olinto MT, Weiderpass E. Pacifier use and short breastfeeding duration: cause, consequence, or coincidence? Pediatrics. 1997;99(3):445-53.

28. American Academy of Pediatrics. Breastfeeding and the use of human milk. Pediatrics. 2005;115(2):496-506.

29. American Academy of Pediatrics. Task Force on Sudden Infant Death Syndrome. The changing concept of sudden infant death syndrome: diagnostic coding shifts, controversies regarding the sleeping environment, and new variables to consider in reducing risk. Pediatrics. 2005;116(5):1245-55.

Recebido em 11 de março de 2009 Modificado em 20 de dezembro de 2009 Aceito em 9 de janeiro de 2010 